\title{
Don't overthink: fast decision making combined with behavior variability perceived as more human-like
}

\author{
S. Marchesi †a,b, J. Perez-Osorio ${ }^{\text {ta }}$, D. De Tommaso ${ }^{a}$, \& A. Wykowska
}

\begin{abstract}
Understanding the human cognitive processes involved in the interaction with artificial agents is crucial for designing socially capable robots. During social interactions, humans tend to explain and predict others' behavior adopting the intentional stance, that is, assuming that mental states drive behavior. However, the question of whether humans would adopt the same strategy with artificial agents remains unanswered. The present study aimed at identifying whether the type of behavior exhibited by the robot has an impact on the attribution of mentalistic explanations of behavior. We employed the Instance Questionnaire (ISQ) pre and post-observation of two types of behavior (decisive or hesitant). We found that decisive behavior, with rare and unexpected "hesitant" behaviors lead to more mentalistic attributions relative to behavior that was primarily hesitant. Findings suggest that higher expectations regarding the robots' capabilities and the characteristics of the behavior might lead to more mentalistic descriptions.
\end{abstract}

\section{Introduction}

Understanding human cognitive processes involved in interactions with artificial agents is crucial for designing robots that are supposed to enter our daily lives. Many studies have focused on how the attribution of human characteristics, like emotions, intentions, or mental states to non-human agents, plays a role in social human-robot interaction [1]. More recently, several studies have examined whether humans can adopt the intentional stance towards humanoid robots $[2,3,4]$.

According to Dennett [5, 6], adopting the intentional stance is a strategy to predict others' behavior with reference to mental states. When we use this strategy, we understand the other agents have their understanding of the world, separate from ours. Importantly, we also assume that those mental states drive people's actions. Dennett distinguishes the intentional stance from the design stance. We use the design stance to understand and predict a device's behavior based on its purpose $[5,6]$. For instance, a kite will fly depending on the strength and direction of the wind, not depending on whether it has the intention to fly.

Humans attribute emotiơns, intentions, and mental states to agents that show some level of autonomous agency. This tendency to anthropomorphize non-human agents has been

$\dagger$ Both authors contributed equally to the paper.

a- Instituto Italiano di Tecnologia

b -University of Manchester

Corresponding author e-mail: jairo.perez-osorio@ iit.it 
considered a default and automatic psychological process [7]. According to Fisher [8], anthropomorphizing could be either interpretative or imaginative. That is, attributing human-like characteristics could be produced by observation of behavior (interpretative anthropomorphism) or by endowing human capabilities to non-human agents by imagining what they can or cannot do (imaginative anthropomorphism). In this context, it is plausible to think that not only experiences or direct observation can produce attribution of humanlike characteristics but also ideas about the capabilities of agents. For example, users can expect a humanoid robot to behave in a humanlike manner even if they have never interacted with a robot. What this theory highlight is that attribution of human-like characteristics to artificial agents might be the result of multiple factors. The physical appearance of the robot, designed to interact with humans, the context, the expectations, and assumptions that one might have regarding the robot and the previous experiences generate attributions of various traits towards the artificial agent.

Social (especially humanoid) robots are an interesting case for examining factors contributing to adopting the intentional stance towards artificial agents. Humanoid robots are machines, but they have a human-like shape, and they are designed to interact with humans. This makes them agents in-between categories, potentially able to elicit the adoption of the intentional stance [9, 10 for review], even though they are just machines. A recent study by Marchesi et al. [2] designed the InStance Questionnaire (ISQ), a novel tool to assess the adoption of the intentional stance towards social robots. The questionnaire was administered online. Respondents observed 34 scenarios depicting the iCub robot [11] and had to choose between two options on how to interpret iCub's behavior. One option described the behavior of the robot with reference to mental states and using a mentalistic vocabulary (intentional/stance). The second one, explained the behavior of the robot in mechanical terms using a technical yocabulary (design stance). Results showed a general tendency to prefer the mechanistic descriptions; however, some mentalistic explanations have also been chosen. Interestingly, some scenarios elicited more mentalistic descriptions than mechanistic ones. The authors concluded that the adoption of the intentional or design stance is context-dependent and might be associated with the depicted behavior. Importantly, these results showed that if people choose to describe the robot behavior in a mentalistic manner in some cases, it means that they are capable in general of adopting the intentional stance towards artificial agents. 
Knowing that people can, in some cases, adopt the intentional stance toward robots, it is important to understand what factors contribute to it. In other words, what are the conditions for adopting the intentional stance. Previous studies have theorized whether people might adopt the intentional stance towards humanoid robots $[10,12,13]$ and even investigated the causality approach towards robot behavior [3]. For example, [3] found that participants used mentalistic explanations to describe the behavior of the robot. Nonetheless, the confidence scores of mentalistic explanations were lower compared to the confidence scores of human actions. This indicates that some degree of cognitive dissonance in describing robots with mentalistic terms. In the case of [2], results showed that some participants were more likely to choose mechanistic scores, while others tended to give more mentalistic explanations. It might be that some participants had certain expectations regarding the human capabilities of the robot and used predominantly mentalistic explanations. In contrast, some other participants had much more mechanistic preassumptions. Perez-Osorio et al. [14] explored this alternative creating a questionnaire that evaluates the expectations of participants regarding iCub. Results showed that people who had high expectations about iCub's behaviors were more prone to explain the behavior of the robot with mentalistic explanations as compared to those who had lower expectations. Participants' expectations and individual differences are one potential factor contributing to the likelihood of adopting the intentional stance. Robot appearance and behavior are obviously other key factors. Ghiglino et al. [15] asked participants to score the human likeness of iCub when it moved its eyes between two fixation points. Slow but variable profile movements yielded higher humanness scores relative to constant speeds. Moreover, human-like eye movements were more engaging and evoked spontaneous attentional following. These findings suggest that the attribution of human-like characteristics might depend on the combination of external factors (subtle variations in social signals) and internal states of the observer, that is, for example, higher-level expectations regarding the behavior of artificial agents.

In the present study, we aimed at evaluating whether the attribution of mechanistic/ mentalistic explanations of robot behavior might be influenced by the type of behavior the robot exhibits, not at the low-level of movement characteristics, but at a level, which might manifest various complex cognitive processes. We designed a paradigm in which two different groups of participants observed a robot performing a cognitive task. Importantly, the robot showed either a predominantly "hesitant" or a predominantly "decisive" behavior 
during a decision-making task. The hesitant behavior took longer to respond and exhibited more body and eye movements compared to the other condition. With the present study, we examined whether being exposed more frequently to a hesitant behavior in a humanoid robot would modulate adoption of the intentional stance. We examined whether the frequency of the hesitant behavior would modulate adoption of the intentional stance. In order to measure the effects of the manipulation, we divided the Instance questionnaire (ISQ [2]) into two parts and administered the first half before the observation of the robot and the other half right after the session with the robot. Additionally, with multiple questionnaires, we explored possible correlations between the ISQ scores, attitudes toward robots, personality traits, and expectations.

\section{Method}

Sample

Forty-two participants (mean age $=24.72, \mathrm{SD}=3.337,19$ men, 4 left-handed) took part in the experiment and received monetary compensation (15€). None of the participants reported a clinical history of psychiatric or neurological diseases. All participants were naïve to the purpose of the experiment and signed written informed consent. At the end of the experimental session, each participant was debriefed by the experimenter. The study was approved by the local Ethical Committee (Comitato Etico Regione Liguria), and it was conducted following the Code of Ethics of the World Medical Association (Declaration of Helsinki).

\section{Procedure}

During the experiment, participants first read and signed the written informed consent. Then, they completed the first group of items (17 out of the 34) from the InStance Questionnaire before the interaction (ISQ PRE), and the RobEx questionnaire [14], Frankenstein Syndrome Questionnaire, FSQ [16], the Negative Attitude Towards Robots questionnaire, NARS [17], and the Robotic Social Attributes Scale, RoSAS [18].

At this point, participants moved inside a cabin where they shared the space with the robot. They sat down in front of iCub and were instructed to observe the behavior of the robot performing a cognitive task. iCub was required to find similarities between three objects presented on the screen. The similarities could be either feature characteristics (like shape or color) or semantic links (same category, like animals). During the task, iCub 
observed two first items presented on the screen and then "had to select" one out of three additional items. The other items were presented vertically and perpendicularly on the right side of the screen (see figure 1, for example). After the robot made a choice, the participants were prompted to respond whether they thought the robot chose the item based on a feature- or category-based link among the items. The type of robot behavior was selected randomly before the beginning of the experiment. Group 1 observed mostly hesitant behavior of the robot while Group 2 observed mainly automatic behavior. After 40 trials, participants proceeded to complete the second half (17 items) from the InStance Questionnaire (ISQ POST), Godspeed questionnaire [19] and Big Five Inventory [20]. Finally, participants were debriefed. Regarding the InStance Questionnaire, participants responded using a slider. On each extreme there was a statement either mentalistic or mechanistic. Participants moved the slider towards one of the sides and were asked to indicate how much that statement explains the robot behavior. The slider scores were converted to a single score, InStance Score (ISS) from o to 100, being o the most mechanistic and 100 the most mentalistic. All the scores in the present study are ISS. The 34 scenarios were randomly selected for every participant and divided into two halves at the beginning of the experiment.

\section{Apparatus and stimuli}

The experiment required two 21" screens placed in the middle of the desk, one facing the robot and the second towards the participant. The displays were tilted back with an angle of $12^{\circ}$ to allow participants to see the robot (see Fig. 1). Participants responded using a standard keyboard. The robot and the experimental presentation of stimuli and data collection were connected to a single workstation (SSD $250 \mathrm{~Gb}, 32 \mathrm{~Gb}$ RAM, Intel i7 7700CPU@3.6o GHz 4 cores, GPU GeForce GTX 1060 3Gb, running Ubuntu 16.04 LTS) through a peer-to-peer Ethernet connection and to both screens with DP cables holding a $100 \mathrm{~Hz}$ refresh rate. The robot and the experiment were controlled using OpenSesame [30], an open-source graphical experimental builder based on Python programming language. Specifically, the iCub robot behaviors have been implemented using the YARP [21] Python wrappers. To command the robot to look at a specific location in space, a 6-DOF gaze controller has been used [22]. The iKinGazeCtrl controller uses inverse kinematics to find eyes and neck positions to make the robot look at specific $3 \mathrm{D}$ Cartesian coordinates. Target locations were predefined so that the poses of the robot were comparable across trials. Regarding the other movements of the robot, specifically the ones related to the hip, we 
used the YARP position controller that generates joints trajectories following the minimum jerk profile. For creating the movement, we added to the predefined positions a uniform random generated displacement useful for making the robot movements slightly different in every trial.

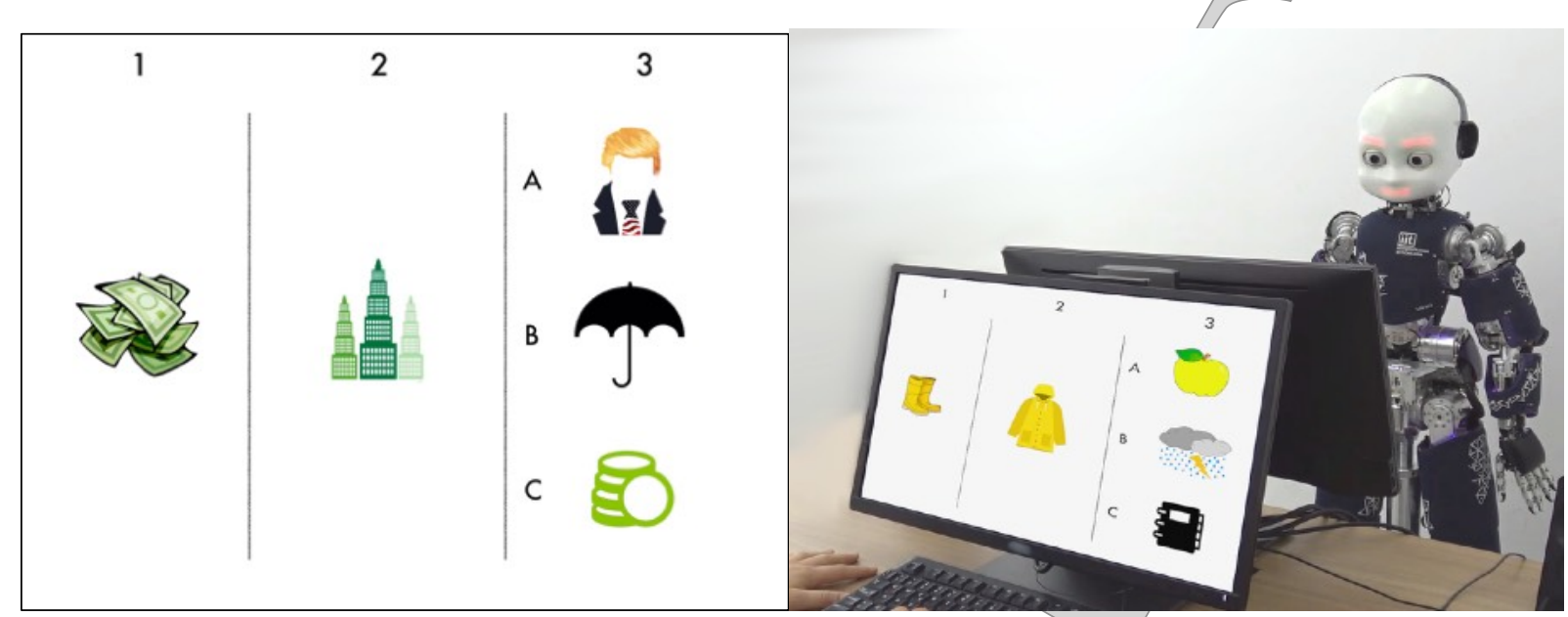

Figure 1. Stimulus example (upper frame) and setup with the robot. (40 scenarios were used on the experiment. The participants saw on the screen the same display presented to the robot).

The items displayed on the screen among which iCub was supposed to choose matching objects were validated in a sample of 86 participants online through the SoSci Survey service. Combinations of items were selected a priori. Participants first saw two items, and from an arrangement of 3 items presented in the right of the screen, they had to choose one that shared either a feature or a categorical connection with the other two, similarly to the task presented in Figure 1. Based on this, we selected the items combinations that were linked by category or by feature by the majority of the participants. We performed a Chisquared analysis on the frequencies of judgment for the category and feature-based links, excluding the catch items, for each combination. Then, we modified the ones that fail to reach significance $(\mathrm{p}=<.05)$, meaning links that were not categorical nor feature-based. Fourteen out of the first 40 combinations, did not reach the significance level and were replaced.

\section{Results}

To measure the impact of the behavior exhibited by the robot on the attribution of mentalistic/mechanistic explanations to the robot, we measured the Instance Questionnaire (ISQ) pre and post-observation in two groups. Results showed that, in general, participants tended to interpret the behavior of the robot in more mechanistic terms $(\mathrm{M}=36.71, \mathrm{SD}=$ 
14.40). However, the comparison between conditions and groups showed an underlying difference. One way ANOVA revealed no significant difference between the groups on the ISQ-PRE (G1-PRE: $\mathrm{M}=33.42, \mathrm{SD}=11.34$; G2-PRE: $\mathrm{M}=35.71, \mathrm{SD}=15.28) ; F(1,40)=.330$, $p=.585$. However, the type of robot behavior had a significant effect on ISQ-POST (G1POST: $\mathrm{M}=34.00, \mathrm{SD}=14.83$; G2-POST: $\mathrm{M}=43.66, \mathrm{SD}=14.52) ; F(1,40)=4.554, p=.039$.

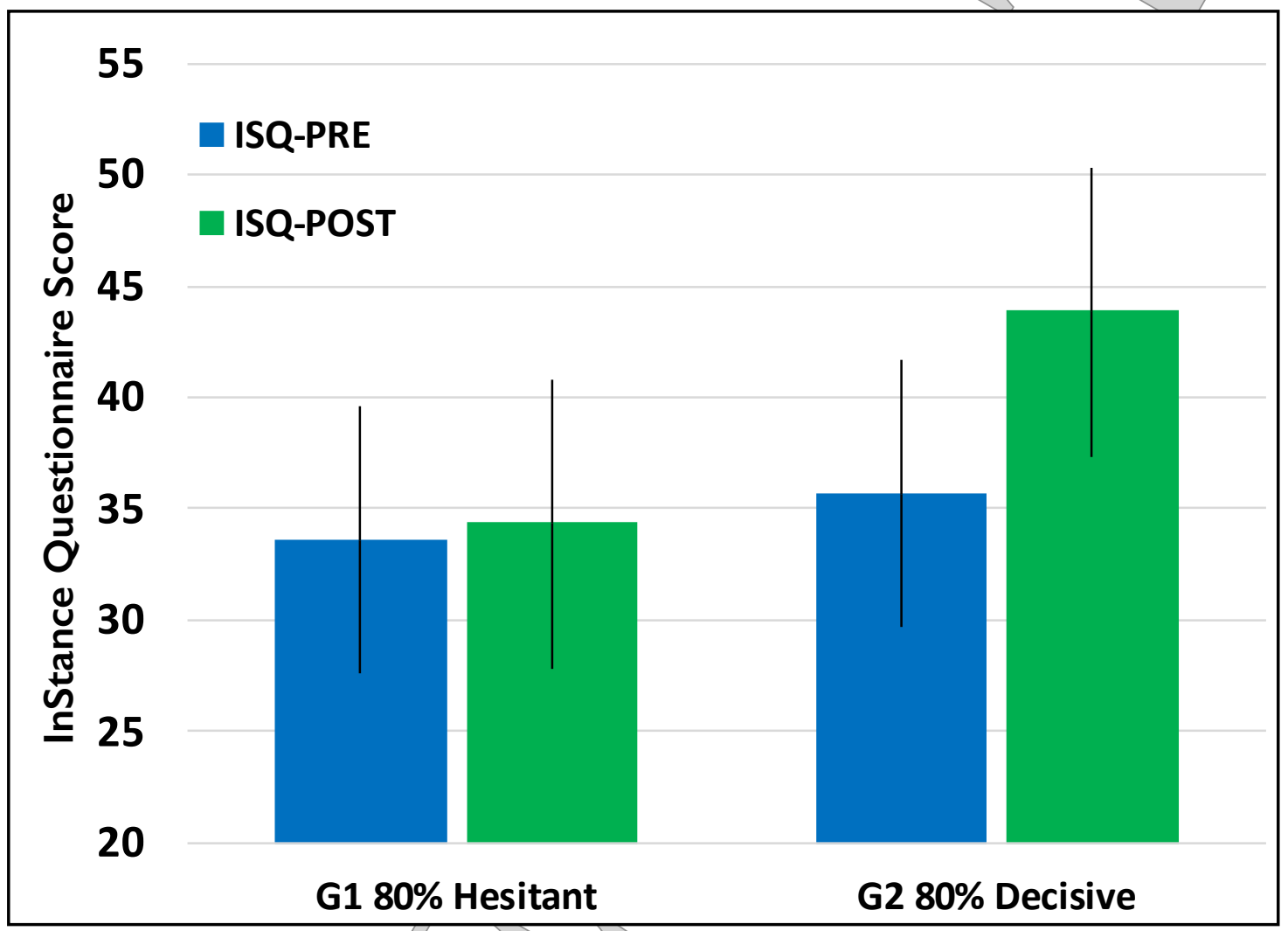

Figure 2. Instance Questionnaire seores for both groups. (The difference between groups on the POST measure was significant. Error bars correspond to the standard error of the mean (SEM)).

This suggests that participants who observed iCub with predominantly automatic behavior had a higher score in the ISQ-POST, compared to the group who observed mainly hesitant behaviors. Within the groups, there were no differences between pre and post $(p>$ .159). Analyses of the additional questionnaires failed to reveal any difference between the groups $(p>$.124). Additionally, we conducted correlations among the different questionnaires subscales and the ISQ-PRE and -POST scores within each group. For Group 1 and Group 2, we found no correlations between ISQ-PRE and the subscales. Correlations were only observed with ISQ-POST. For Group 1, only a positive correlation between ISQPOST and Godspeed Anthropomorphism scale $[r(21)=.550, p=.020]$ was observed. However, for Group 2, several correlations were found for the ISQ-POST: on the positive 
direction with GS Anthropomorphism subscale $[r(21)=.496, p=.022]$, GS Animacy subscale $[r(21)=.473, p=.030]$, RoSaS Competence subscale $[r(21)=.490, p=.024]$, BFI Conscientiousness subscale $[r(21)=.436, p=.048]$, FSQ expectations subscale $[r(21)=.534$, $p=.013]$ and finally a negative correlation for the RoSaS discomfort subscale $[r(21)=-.509$, $p=.018]$.

Table 1. Correlations between Instance questionnaire scores and other questionnaires.

\begin{tabular}{|c|c|c|c|c|c|c|c|c|}
\hline & \multicolumn{4}{|c|}{ Group 1: more hesitant } & \multicolumn{4}{|c|}{ Group 2: more Decisive } \\
\hline & PRE & $p$ & POST & $P$ & PRE & & OST & $p$ \\
\hline IQS-PRE & 1 & & .307 & .175 & 1 & & 7.399 & .073 \\
\hline IQS-POST & .307 & .175 & 1 & & .399 & .073 & 1 & \\
\hline GS Ant & -.274 & .229 & $.503^{*}$ & .020 & .065 & .779 & $.496^{*}$ & .022 \\
\hline GS Ani & -.223 & .332 & .278 & .222 & .151 & .514 & $.473^{*}$ & .030 \\
\hline GS Like & -.029 & .900 & .385 & .085 & .093 & 687 & .256 & .262 \\
\hline FSQ Exp & .366 & .103 & .280 & .219 & -.028 & .905 & $.534^{*}$ & .013 \\
\hline FSQ Anx & -.432 & .051 & .049 & .832 & -.150 & .517 & -.215 & .349 \\
\hline FSQ SocRis & -.288 & .206 & -.188 & 415 & -.238 & .298 & -.163 & .480 \\
\hline FSQ Trust & .097 & .676 & -.195 & .397 & & .490 & .369 & .100 \\
\hline NARS Emo & .132 & .568 & .070 & .762 & -.256 & .262 & .381 & .088 \\
\hline NARS Sit & -.202 & .381 & .206 & .371 & -.140 & .544 & -.425 & .055 \\
\hline NARS Soc & -.429 & .052 & .220 & .339 & -.131 & .571 & -.113 & .626 \\
\hline RoSASCom & .212 & .356 & .030 & .898 & -.165 & .474 & $.490^{*}$ & .024 \\
\hline RoSAS Dis & -.291 & .200 & -.058 & .802 & -.176 & .445 & $-.509^{*}$ & .018 \\
\hline RoSASWar & .275 & .227 & .431 & .051 & -.138 & .552 & .353 & .116 \\
\hline BFI Extra & -.040 & .865 & -.116 & .617 & .419 & .059 & .211 & .358 \\
\hline BFI Agree & -.055 & .811 & -.181 & .433 & .308 & .174 & .177 & .443 \\
\hline BFI Cos & .247 & .280 & .060 & .797 & -.251 & .273 & $.436^{*}$ & .048 \\
\hline BFI Neuro & -.051 & 828 & .117 & .614 & -.372 & .096 & -.264 & .248 \\
\hline BFI Open & -.032 & .890 & -.333 & .140 & -.097 & .676 & .410 & .065 \\
\hline
\end{tabular}

Interestingly, among the subscales, we found that the FSQ Expectations score had a strong positive correlation with GS Anthropomorphism subscale $[r(21)=.730, p>.000]$, GS Animacy subscale $[r(21)=.760, p>.000]$, RoSaS Competence subscale $[r(21)=.624, p$ $=.003]$. We conducted a post-hoc ANCOVA analysis to compare the ISQ-POST whilst controlling for expectations. Levene's test and normality checks were carried out, and the 
assumptions met. There was a significant difference between groups $[F(1,39)=5.047, p=$ .03, partial Eta squared .115]. The effect of FSQ expectations scores was also significant $[F(1,39)=8.202, p=.007$, partial Eta squared .174$]$ This suggests that differences observed between groups are influenced, but not entirely depending on participants expectations.

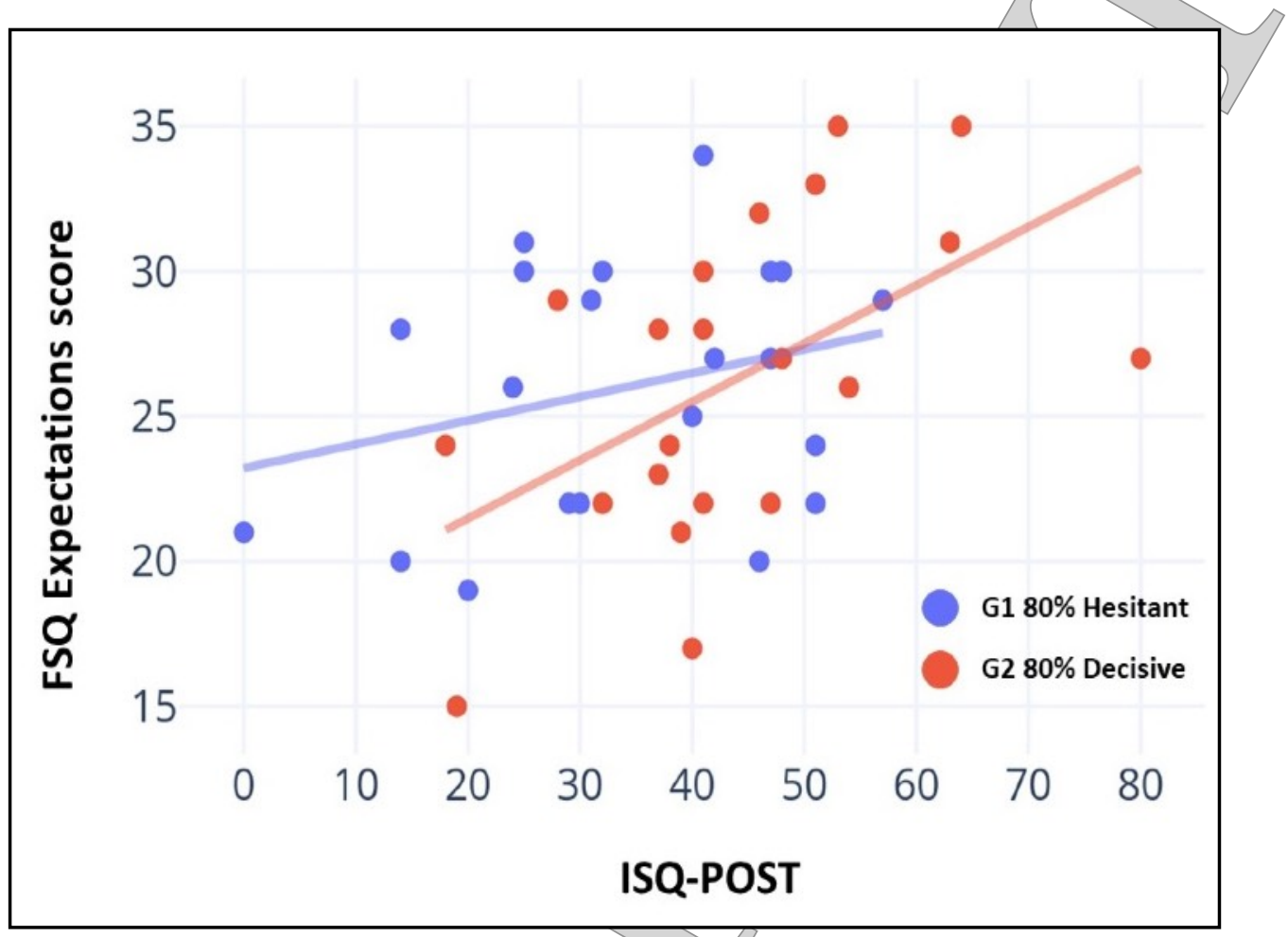

Figure 3. Scatter plot and trend lines between FSQ expectations score and ISQ-POST (Participants that had higher scores on the FSQ subscale tended to score higher on ISQ-POST. This difference was significant for Group 2 in red).

\section{Discussion}

The present study aimed at evaluating whether a behavior manifesting cognitive processes, exhibited by a humanoid robot has an impact on the attribution of mentalistic/ mechanistic explanations. We found that scores for the group of participants who observed a robot exhibiting a decisive behavior on a decision-making task were higher (more mentalistic) relative to the scores of the group that observed the hesitant behavior. Similar to previous findings, and despite the difference in the scores between groups, all the participants judged the behavior of the robot mainly in mechanistic terms with tendencies towards the middle of the scale. Additionally, we found significant correlations between multiple subscales of various questionnaires and the POST-ISQ score including: Godspeed Anthropomorphism and Animacy, RoSaS competence, FSQ Expectations subscale, and BFI 
Conscientiousness, and an additional inverse correlation with RoSaS discomfort. Our results suggest that participants were sensitive to robot behavior, which made a difference on the attribution of mechanistic explanations on the InStance questionnaire after observation of behavior.

Interestingly, people were more likely to attribute mental capabilities to iCub performing a predominantly decisive behavior and a small percentage of hesitations. These results might seem at first counter-intuitive, as we might expect that less variable behavior would elicit less mentalistic explanations. However, it is expected that decisive behavior and some degree of variability (hesitant behavior) might have been perceived as more human-like than the robot that displayed consistently hesitant actions. Literature has shown that people expect robots to be precise, efficient, competent, and reliable with some degree of social skills [23]. This was also revealed on the scales we applied before the experiment. Thus, participants' expectations fit better with a fast responding and competent robot. Simultaneously, the lower probability of occurrence of the hesitant behavior, and thus high degree of unexpectancy, might have surprised the observers and made a more human-like impression, translating on higher scores for the group that observed this behavior. We argue that when hesitant behavior was "the rule", rather than "exception", it lost the surprise factor and became a delayed and not efficient behavior and was not interpreted as the result of mental capacities but rather as a hindrance to performance. Therefore, such robot might evoke less impressions of an intentional agent.

In addition, the small amount of hesitant behaviors might have been interpreted as a vulnerability, revealing that the robot was not able to complete the task $100 \%$ of the time. Studies have shown that when people perceive the robot as vulnerable, they feel more comfortable during interactions and tend to include the robot more in-group dynamics, relative to robots that do not recognize having difficulties in completing tasks [24]. This might have made the robot appear more human-like, and thus described in terms that are more mentalistic. However, this effect might break when vulnerability becomes a rule and impedes the robot to perform a task.

Regarding the results from the questionnaires, it is worth mentioning that both groups had very similar profiles before and after the experiment in terms of expectations and attitudes towards robots. However, after observation, the only observable difference between groups was the ISQ-POST, which suggests that in order to elicit mentalistic 
explanations to artificial agents' behavior, a general positive predisposition towards robots might be required, but it is not sufficient. ISQ-POST scores were also correlated to Conscientiousness scores on the Big Five Inventory. This subscale evaluates the personality trait linked to being organized, efficient, and diligent. Our results might suggest that an artificial agent that reflects certain personality characteristics might be perceived as more human-like. In our case, additionally to the mentioned factors, the observed behavior might have resonated with peoples' preferences and thus was judged as a result of internal states. Individual differences might be crucial when adopting the intentional stance. Further studies should address this phenomenon.

We observed that the likelihood of using mentalistic explanations to describe iCub's behavior was influenced principally by the type of behavior observed. However, we investigated whether this difference could have been attributed to participants' expectations regarding robots' general capabilities, according to the Expectations scores on the FSQ questionnaire. We found that although expectations play a role in the difference of scores, the main effect was associated with the observation of a decisive behavior. Some studies have pointed out that the discrepancy between expectations and reality might affect humanrobot interaction $[13,14,25,26,27]$. These studies indicate that human-like robots elicit more elaborated expectations regarding competence, cognition, social interaction skills and emotions, relative to more machine-like platforms. Therefore, design and behavioral elements should be aligned with the agent's performance. We speculate that expectations may also play a role in the attribution of mental states to artificial agents during social interaction. If those expectations match observed behavior, humans might be more likely to use mentalistic explanations. Future studies should deepen the knowledge regarding the link between expectations and the attribution of mental states to artificial agents.

\section{Conclusion}

The present paper addressed the question of relationship between observed behavior and attribution of mental states to a humanoid robot. We suggest that variations of behavior reflecting cognitive processes exhibited by a humanoid robot modulate the use of mentalistic explanations. Interestingly, decisive behavior with a sporadic and unexpected "hesitant" behavior was related to mentalistic attributions. Although expectations regarding the performance of the robot play a role in the mental attribution, the kind of behavior exhibited has more considerable weight on the adoption of the intentional stance. 
Our results also suggest that individual differences might be taken into account on the interpretation of human-robot interaction findings.

\section{Acknowledgments}

This work received support from the European Research Council (ERC) under the European Union's Horizon 2020 research and innovation program (grant awarded to AW, titled "InStance: Intentional Stance for Social Attunement." G.A. No: ERC-2016StG-715058). The content of this paper is the sole responsibility of the authors. The European Commission or its services cannot be held responsible for any use that may be made of the information it contains.

\section{References}

1. N. Epley, A. Waytz, and J. T. Cacioppo, "On Seeing Human: A Three-Factor Theory of Anthropomorphism," Psychol. Rev., 2007.

2. S. Marchesi, D. Ghiglino, F. Ciardo, J. Perez-Osorio, E. Baykara, and A. Wykowska, "Do we adopt the intentional stance/toward humanoid robots?," Front. Psychol., 2019.

3. S. Thellman, A. Silvervarg, and T. Ziemke, "Folk-psychological interpretation of human vs. humanoid robot behavior: Exploring the intentional stance toward robots," Front. Psychol., 2017.

4. S. Thellman, A. Giagtzidou, A. Silvervarg, and T. Ziemke, "An implicit, non-verbal measure of belief attribution to robots," in ACM/IEEE International Conference on Human-Robot Interaction, 2020.

5. $\quad$ D. C. Dennett, "Intentional systems," J. Philos., vol. 68, no. February, 1971.

6. Dennett, D. C. (1989). The intentional stance. MIT press.

7. R.W. Mitchell, N. S. Thompson, H. L. Miles, "Anthropomorphism, Anecdotes, and Animals," Current Anthropology. 1997.

8. J. A. Fisher. "Disambiguating anthropomorphism: An interdisciplinary review." Perspectives in ethology 9, no. 4. 1991.

9. J. Perez-Osorio and A. Wykowska, "Adopting the intentional stance towards humanoid robots," in Springer Tracts in Advanced Robotics, 2019.

10. J. Perez-Osorio and A. Wykowska, "Adopting the intentional stance toward natural and artificial agents," Philosophical Psychology. 2020. 
11. G. Metta et al., "The iCub humanoid robot: An open-systems platform for research in cognitive development," Neural Networks, 2010.

12. H. L. Gallagher, A. I. Jack, A. Roepstorff, and C. D. Frith, "Imaging the intentional stance in a competitive game," Neuroimage, vol. 16, pp. 814-821, 2002.

13. T. Chaminade et al., "How do we think machines think? An/ MRI study of alleged competition with an artificial intelligence," Front. Hum. Neurosci., vol. 6, no. MAY 2012, pp. 1-9, 2012.

14. J. Perez-Osorio, S. Marchesi, D. Ghiglino, M. Ince, and A. Wykowska, "More Than You Expect: Priors Influence on the Adoption of Intentional Stance Toward Humanoid Robots," in Lecture Notes in Computer Science (including subseries Lecture Notes in Artificial Intelligence and Lecture Notes in Bioinformatics), 2019.

15. D. Ghiglino, C. Willemse, D. De Tommaso, F. Bossi, and A. Wykowska, "At first sight: Robots' subtle eye movement parameters affect human/attentional engagement, spontaneous attunement and perceived human-likeness," Paladyn, 2020.

16. T. Nomura, D. S. Syrdal, and K. Dautenhahn, "Differences on Social Acceptance of Humanoid Robots between Japan and the UK," 4th Int. Symp. New Front. HumanRobot Interact., 2015.

17. D. S. Syrdal, K. Dautenhahn, K. L. Koay, and M. L. Walters, "The negative attitudes towards robots scale and reactions to robot behaviour in a live human-robot interaction study," Adapt. Emergent Behav. Complex Syst., 2009.

18. C. M. Carpinella, A. B. Wyman, M. A. Perez, and S. J. Stroessner, "The Robotic Social Attributes Scale (RoSAS)," in Proceedings of the 2017 ACM/IEEE International Conference on Human-Robot Interaction - HRI '17, 2017.

19. C. Bartneck, D. Kulić, E. Croft, and S. Zoghbi, "Measurement instruments for the anthropomorphism, animacy, likeability, perceived intelligence, and perceived safety of robots," Int. J. Soc. Robot. 2009.

20. S. S. John, O. P., "Big Five/Inventory ( Bfi )," in Handbook of Personality Second Edition: Theory and Research, 1999.

21. G. Metta, P. Fitzpatrick, and L. Natale, "YARP: Yet Another Robot Platform," Int. J. Adv. Robot. Syst., Mar. 2006, vol. 3, no. 1, p. 8.

22. A. Roncone, U. Pattacini, G. Metta, and L. Natale, "A Cartesian 6-DoF Gaze Controller for Humanoid Robots," Proceedings of Robotics: Science and Systems, 2016. 
23. A. C. Horstmann and N. C. Krämer, "Great expectations? Relation of previous experiences with social robots in real life or in the media and expectancies based on qualitative and quantitative assessment," Front. Psychol., 2019.

24. S. Strohkorb Sebo, M. Traeger, M. Jung, and B. Scassellati, "The Ripple Effects of Vulnerability: The Effects of a Robot's Vulnerable Behavior on Trust in Human-Robot Teams," in ACM/IEEE International Conference on Human-Robot Interaction, 2018.

25. T. Komatsu, R. Kurosawa, and S. Yamada, "How Does the Difference Between Users' Expectations and Perceptions About a Robotic Agent Affect Their Behavior?: An Adaptation Gap Concept for Determining Whether Interactions Between Users and Agents Are Going Well or Not,” Int. J. Soc. Robot, 2012.

26. L. T. Schramm, D. Dufault, and J. E. Young, "Warning: This robot is not what it seems! exploring expectation discrepancy resulting from robot design," in ACM/IEEE International Conference on Human-Robot Interaction, 2020.

27. C. Edwards, A. Edwards, P. R. Spence, and D. Westerman, "Initial Interaction Expectations with Robots: Testing the Human-To-Human Interaction Script," Commun. Stud., 2016.

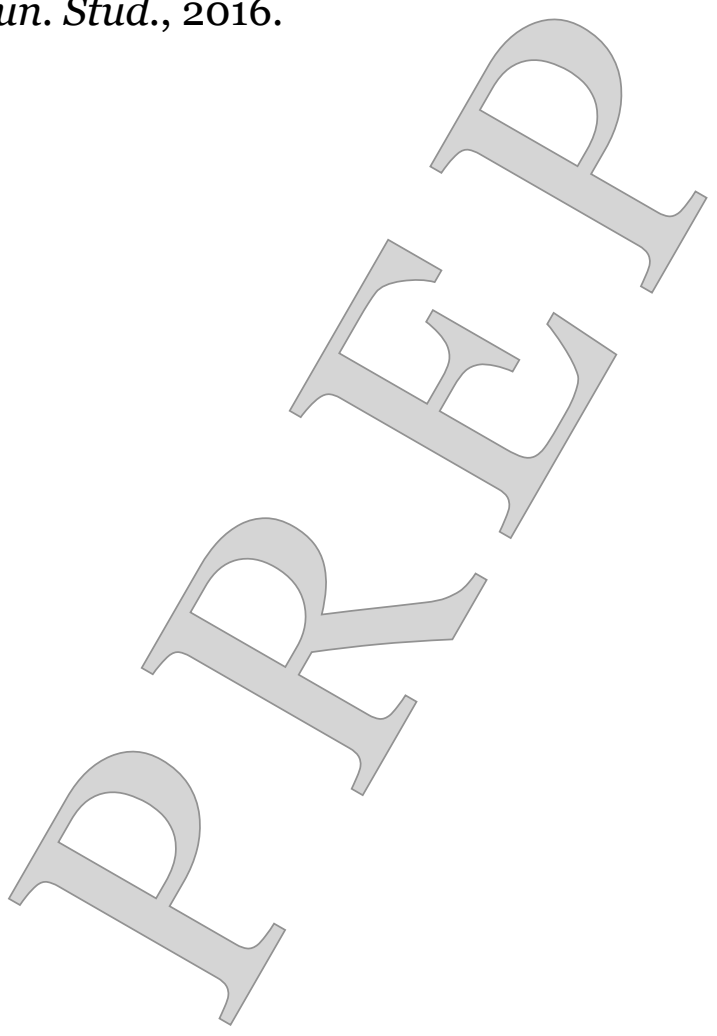

